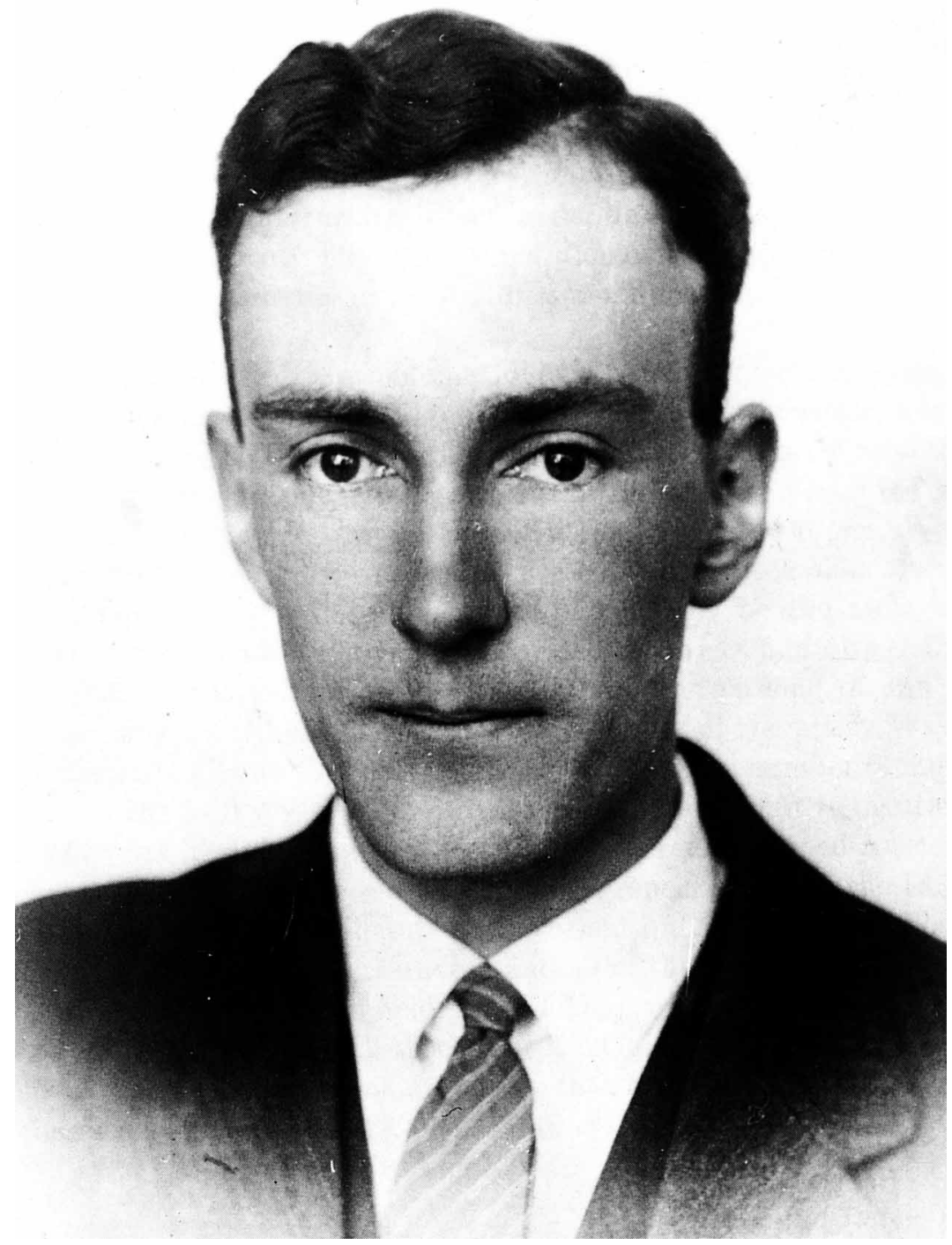

Dr. J.H. Oort.

"Courtesy Sterrewacht Leiden" 


\title{
OORT IN MEMORIAM
}

\author{
ADRIAAN BLAAUW \\ Kapteyn Laboratory, University of Groningen
}

Jan Hendrik Oort died in November 1992 after a life devoted to astronomy. A life that brought him, and his beloved wife Mieke, the happiness of family life and innumerable honours, and the affection and admiration of colleagues all over the world. It is fitting that we devote this Symposium to the memory of a man to whom students of galactic research owe more, I think, than to any other astronomer of the 20th century.

Oort studied at Groningen under J.C. Kapteyn, whose approach to astronomical research has deeply influenced Oort's own work: always aiming at close confrontation of theory with observation, with a critical look at the real value of the observations, and keeping the theoretical treatment as transparent as possible. And always alert, with an open eye for that unexpected, easily overlooked feature that might contain the clue for opening a new field of interest. He was a man of extraordinary perception and perseverance.

If I ask youngest astronomers about Oort's work, the chances are that they will mention him as the discoverer of the "Oort Cloud" of comets; this has been referred to frequently in relation to recent, widely publicized events. If I ask the generation that entered astronomy in the 1960s and 1970s, they will mention his pioneering work in radioastronomy: the establishment of the radio observatories at Dwingeloo and Westerbork, the 21-cm surveys of the Galaxy, and subsequent extragalactic work. My own first acquaintance with Oort's work dates from still farther back: the mid-1930s, less than a decade after Oort's fundamental work on differential galactic rotation and the implications for the dimensions and dynamics of the Galaxy - and I 
subsequently witnessed his interest shifting to the role of the interstellar medium in the context of galactic and extragalactic structure in the 1940s and 1950s. For me, this first phase of Oort's career has always remained the most impressive. All he did arose from his deepest motivation: curiosity about the structure and history of the all-embracing Universe.

Apart from his pure research, we owe to Oort initiatives that led to some of the most impressive facilities now accessible to the observational astronomer: the radio observatories at Dwingeloo and Westerbork and the European Southern Observatory. And we remember how deeply he has inspired the work of new generations of astronomers with these radioastronomical facilities!

For those of us who have been privileged to be associated with Jan Oort, be it as student or as a colleague, there will remain the long lasting memory of a man of extraordinary leadership and inspiration, and of a dearly loved friend. 